\title{
QoS-driven Runtime Adaptation of Service Oriented Architectures
}

\author{
Valeria Cardellini $^{1} \quad$ Emiliano Casalicchio ${ }^{1} \quad{\text { Vincenzo } \text { Grassi }^{1}}^{1}$
}

\author{
Francesco Lo Presti ${ }^{1}$ \\ Raffaela Mirandola ${ }^{2}$ \\ ${ }^{1}$ Dipartimento di Informatica, Sistemi e Produzione, Università di Roma "Tor Vergata", 00133 Roma, Italy \\ \{cardellini, casalicchio, vgrassi, lopresti\}@disp.uniroma2.it \\ ${ }^{2}$ Dipartimento di Elettronica e Informazione, Politecnico di Milano, 20133 Milano, Italy \\ mirandola@elet.polimi.it
}

\begin{abstract}
Runtime adaptation is recognized as a viable way for a serviceoriented system to meet QoS requirements in its volatile operating environment. In this paper we propose a methodology to drive the adaptation of such a system, that integrates within a unified framework different adaptation mechanisms, to achieve a greater flexibility in facing different operating environments and the possibly conflicting QoS requirements of several concurrent users. To determine the most suitable adaptation action(s), the methodology is based on the formulation and solution of a linear programming problem, which is derived from a behavioral model of the system updated at runtime by a monitoring activity. Numerical experiments show the effectiveness of our approach. Besides the methodology, we also present a prototype tool that implements it.
\end{abstract}

\section{Categories and Subject Descriptors}

D.2.11 [Software Engineering]: Software Architectures

; C.4 [Performance of Systems]: Modeling Techniques

\section{General Terms}

Management, Performance, Reliability

\section{Keywords}

Service-oriented architecture, runtime adaptation, quality of service.

\section{INTRODUCTION}

Service Oriented architecture (SOA) is emerging as a paradigm for the design of complex software systems, which encourages the realization of new software systems by composing network-accessible loosely-coupled services. According to this paradigm, the development focus shifts from activities concerning the in-house custom

Permission to make digital or hard copies of all or part of this work for personal or classroom use is granted without fee provided that copies are not made or distributed for profit or commercial advantage and that copies bear this notice and the full citation on the first page. To copy otherwise, to republish, to post on servers or to redistribute to lists, requires prior specific permission and/or a fee.

ESEC-FSE'09, August 23-28, 2009, Amsterdam, The Netherlands. Copyright 2009 ACM 978-1-60558-001-2/09/08 ...\$10.00. design and implementation of the system components, to activities concerning the identification, selection, and composition of services offered by third parties.

These activities are driven by the goal of fulfilling both the functional requirements concerning the overall business logic that must be implemented and the non-functional requirements concerning the quality of service (QoS) levels that should be guaranteed by the system.

Achieving this goal in a SOA environment is a challenging task, mainly because of its dynamic and unpredictable nature. To cope with this problem, a generally accepted idea is that a SOA system should be able to dynamically self-adapt to the changing conditions of its operating environment. Several methodologies have been proposed in the literature for this purpose (e.g., [2, 5, 15, 20, $21,26]$ ), concerning the fulfillment of functional or non-functional requirements. In this paper, we present MOSES (MOdel-based SElf-adaptation of SOA systems), a methodology and a prototype tool aimed at driving the self-adaptation of a SOA system to fulfill non-functional QoS requirements such as system performance, reliability, and cost.

Devising QoS-driven adaptation methodologies of SOA systems is of utmost importance in the envisaged service marketplace, where different services may co-exist implementing the same functionality (we refer to the former as concrete services and the latter as abstract service). These competing services are differentiated by their QoS, thus allowing a prospective user to choose the services that best suit his/her QoS requirements. Hence, being able to deliver and guarantee the QoS level required by a given class of users may bring competitive advantage to a service provider over other providers. In this respect, it is generally assumed in the SOA environment that the service provider and user engage in a negotiation process, which culminates in the definition of a Service Level Agreement (SLA) concerning their respective obligations and expectations about QoS [21].

Most of the proposed methodologies for QoS-driven adaptation of SOA systems address this problem as a question concerning which services should be used (service selection problem): given the set of abstract services needed to compose a new added value service, the goal is to identify for each abstract service a corresponding single concrete service, selecting it from a set of candidates (e.g., [2, 5, 7, 11, 21, 27]). When the operating conditions change (e.g., a selected concrete service is no longer available, or its delivered QoS has changed, or the user QoS requirements have changed), a new selection can be calculated and the abstract ser- 
vices which compose the SOA system are dynamically bound to a new set of concrete services.

However, it may happen that, under a specific operating condition, no selection exists of single concrete services allowing the fulfillment of the QoS requirements. In this case, adaptation methodologies based only on service selection fail to meet their objective, which could cause a loss of income and/or reputation for a service provider.

To overcome this problem, with MOSES we propose to broaden the range of the considered adaptation mechanisms, by exploiting the availability in a SOA environment of multiple independent implementations of the same functionality. Rather than considering only the binding of each abstract service to one single concrete service that implements it, MOSES can more generally bind each abstract service to $a$ set of functionally equivalent concrete services, coordinated according to some spatial redundancy pattern (e.g., 1out-of-n redundancy, sequential retry). In this way, MOSES may achieve QoS levels (concerning reliability and, in some cases, performance) that could not be achieved otherwise, thus increasing the flexibility of a provider in facing a broader range of QoS requirements and volatile operating conditions. The service selection mechanism considered in other papers thus becomes a special case of this more general mechanism, when the cardinality of the identified set of concrete services is equal to one. Of course the advantage achieved in this way should be weighed against the higher cost caused by the use of multiple concrete services.

Hence, the basic idea underlying MOSES is that, besides asking which concrete services should be bound to the abstract services used to compose a SOA system, we should also ask how to use them to meet the QoS requirements.

To achieve the adaptation goal, the MOSES framework relies on the use of a suitable model of the SOA system to be adapted, which is kept up to date at runtime by a monitoring activity. This model is dynamically used to calculate a re-arrangement of the available concrete services (i.e., a new answer to the which and how questions), to use at best these services each time a relevant change is detected in the operating environment. The appropriate adaptation actions used at runtime by MOSES are determined by solving a Linear Programming optimization problem, whose formulation allows us to efficiently cope with a fast changing operating environment.

The remainder of the paper is organized as follows. In Section 2 we present an overview of the MOSES framework, which is then detailed in the next sections. In Section 3 we describe how we model within the MOSES framework an adaptable SOA system, and the related model that MOSES maintains at run time to support the application of the adaptation methodology. Based on these models, in Section 4 we present the formulation of an optimization problem that is solved within the MOSES framework. Then, in Section 5 we present the prototype tool that implements our methodology, and numerical experiments that illustrate the kind of adaptation directives issued by MOSES. In Section 6 we discuss related works. Finally, in Section 7 we give some conclusions and suggestions for future work.

\section{THE MOSES APPROACH}

Devising an adaptation methodology strongly depends on the assumptions made about the scenario where it will operate. Different assumptions may lead to different formulations of the problem to be solved, and corresponding solution methodologies.

In this respect, MOSES focuses on a scenario where several users address a relatively sustained traffic of requests to a SOA system architected as a composite service. Each user may have its own QoS requirements, and negotiates a corresponding SLA with the system. In this scenario, we assume that the QoS requirements stated in the SLA concern the average value of QoS attributes calculated over all the requests belonging to a flow generated by a given user.

Within this scenario, the goal of MOSES is to dynamically adapt the implementation of the SOA system it manages, to meet the (possibly conflicting) per-flow QoS requirements of several users. Figure 1 shows the core elements of the MOSES framework, their overall organization and their mapping onto the Monitor-AnalyzePlan-Execute (MAPE) model of adaptation proposed within the Autonomic Computing framework [16]. In this perspective, MOSES can be seen as an instantiation of this model for the SOA domain.

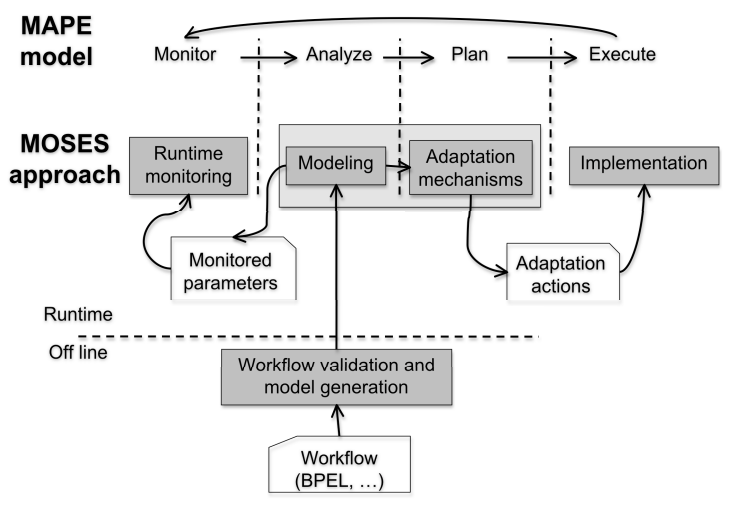

Figure 1: The MOSES approach.

The MOSES input consists of the description of the composite service in some suitable workflow orchestration language (e.g., BPEL [22]), and the set of candidate concrete services implementing the required functionalities (including the parameters of their SLAs). At present, MOSES relies on external entities for the definition of the workflow, the identification of the candidate concrete services and the negotiation with them of suitable SLAs.

The received workflow description is checked against a model of the class of SOA systems MOSES is able to manage, to verify whether it belongs to this class. In the positive case, MOSES builds a behavioral model of the composite service, and passes it to the runtime adaptation modules.

As a first step to carry out runtime adaptation, MOSES uses the behavioral model to build the template of an optimization problem. The parameters values for this problem are derived from the parameters of the SLAs negotiated with the composite service clients and providers, and from a monitoring activity. As detailed in Section 5, this latter activity is partly implemented within MOSES itself, and partly relies on third party services.

Besides maintaining up to date the parameters of the optimization problem, the monitoring activity checks and notifies whether some relevant change occurs in the composite service environment. Changes to be tracked include the arrival/departure of a client with the associated SLA, an observed variation in the SLA parameters of the constituent concrete services, and the addition/removal of a concrete service from the set of available concrete services.

Upon receiving from the monitoring service a notification of a significant variation of the model parameters, MOSES finds out whether an adaptation action must be performed. To this end, it 
builds a new instance of the optimization problem, with the new values of the parameters. The calculated solution provides indications about the adaptation actions that must be performed to optimize the use of the available resources (i.e., the concrete services) with respect to some utility criterion, within the constraints defined by the existing SLAs. Based on this solution, MOSES issues suitable directives to its implementation module, so that future concrete instances of the composite service workflow will be generated according to these directives. We detail in Sections 3 and 4 the kind of directives generated by MOSES, and in Section 5 a prototype implementation.

\section{MODELING OF AN ADAPTABLE SOA SYSTEM}

\subsection{Architectural Model}

We use a "two level" grammar to model the kind of SOA system MOSES refers to, and to illustrate some basic concepts of the MOSES methodology ${ }^{1}$.

The two levels of the grammar reflect a separation of concerns between two different roles: the first level concerns the role of composition manager, whose goal is the specification of the business process. Within MOSES, this level is only used to check whether the orchestration pattern of an actual SOA system matches the kind of patterns MOSES is able to manage and, in the positive case, to support the construction of a suitable model. Other issues concerning this first level are out of the MOSES scope.

The second level concerns the role of adaptation manager, whose goal (in our approach) is to determine for each abstract service, which concrete services should be used to implement it and how they should be used, to meet the QoS requirements in a volatile operating environment. MOSES specifically focuses on this second role and its implementation in a SOA system.

The first level is defined as follows:

$$
\begin{aligned}
& \mathrm{C}::=\mathrm{S}\left|\operatorname{seq}\left(\mathrm{C}^{+}\right)\right| \operatorname{loop}(\mathrm{C})\left|\operatorname{sel}\left(\mathrm{C}^{+}\right)\right| \text {par_and }\left(\mathrm{C}^{+}\right) \\
& \mathrm{S}::=S_{1}\left|S_{2}\right| \ldots \mid S_{m}
\end{aligned}
$$

In this definition, C denotes a composite service, $S_{1}, S_{2}, \ldots, S_{m}$ denote abstract services (i.e., functionalities needed to compose a new added value service), and $\mathrm{C}^{+}$denotes a list of one or more services. Hence, MOSES currently encompasses composite services consisting either of a single service, or of the orchestration of other services according to the composition rules: seq, loop, sel, par_and. Table 1 summarizes the intended meaning of these rules. We point out that the above grammar is purposely abstract, as it intends to specify only the structure of the considered composite services. Details such as how to express the terminating condition for a loop are therefore omitted. The grammar does not capture all the possible structured orchestration patterns (a broader set is presented, for example, in [4]), but includes a significant subset. Figure 2 shows an example of orchestration pattern described as a UML2 activity diagram, and the corresponding instance generated by the first level of the grammar.

The second level consists of a set of production rules for each abstract service $S_{i}$ considered at the first level $(1 \leq i \leq m)$, defined

\footnotetext{
${ }^{1}$ We point out that our use of the term "two level" grammar has no relationship with the homonimous concept used in formal language theory (e.g., Van Wijngaarden grammar).
}

Table 1: Workflow composition patterns.

\begin{tabular}{|c|l|}
\hline Rule & \multicolumn{1}{c|}{ Meaning } \\
\hline $\operatorname{seq}\left(\mathrm{C}^{+}\right)$ & sequential execution of the services in $\mathrm{C}^{+}$ \\
\hline loop $(\mathrm{C})$ & repeated execution of the service C \\
\hline sel $\left(\mathrm{C}^{+}\right)$ & conditional selection of one service in $\mathrm{C}^{+}$ \\
\hline par_and $\left(\mathrm{C}^{+}\right)$ & $\begin{array}{l}\text { concurrent execution of the services in } \mathrm{C}^{+} \\
\text {(with complete synchronization) }\end{array}$ \\
\hline
\end{tabular}

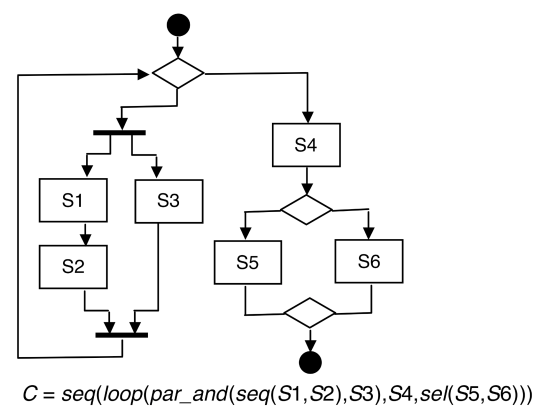

Figure 2: A MOSES-compliant workflow.

as follows:

$$
\begin{aligned}
S_{i}::=\operatorname{switch}\left(P_{i}^{+}\right) \\
P_{i}::=\operatorname{single}\left(K_{i}\right)\left|\operatorname{alt}\left(K_{i}^{+}\right)\right| \text {par_or }\left(K_{i}^{+}\right) \\
K_{i}::=k_{i 1}\left|k_{i 2}\right| \ldots \mid k_{i n_{i}}
\end{aligned}
$$

In this definition, $k_{i 1}, k_{i 2}, \ldots, k_{i n_{i}}$ denote functionally equivalent concrete services that have been identified as candidates to implement the abstract service $S_{i}$, and $K_{i}^{+}$is an ordered list of one or more of such concrete services. If $K_{i}^{+}$consists of more than one concrete service, these services must be coordinated according to a coordination pattern $P_{i}$. More than one pattern can be used to get an overall implementation of $S_{i}$. Hence, the implementation of $S_{i}$ consists in general of a switch that, for each invocation of $S_{i}$, selects one of the patterns listed in $P_{i}^{+}$.

At present, the MOSES framework includes three coordination patterns, denoted as single, alt and par_or. Table 2 summarizes their intended meaning. Besides the simple single pattern (where a single concrete service implements $S_{i}$ ), the other two patterns use multiple concrete services to improve some QoS attributes of the $S_{i}$ implementation with respect to the single pattern, as it will be explicitly shown in the next subsection.

Table 2: Coordination patterns.

\begin{tabular}{|c|l|}
\hline Rule & \multicolumn{1}{c|}{ Meaning } \\
\hline single $\left(K_{i}\right)$ & execution of a single concrete service \\
\hline alt $\left(K_{i}^{+}\right)$ & $\begin{array}{l}\text { sequential (alternate) execution of the concrete } \\
\text { services listed in } K_{i}^{+}, \text {until either one of them } \\
\text { successfully completes, or the list is exhausted }\end{array}$ \\
\hline par_or $\left(K_{i}^{+}\right)$ & $\begin{array}{l}\text { concurrent execution of the concrete services in } \\
K_{i}^{+} \text {(with 1 out of } n \text { synchronization) }\end{array}$ \\
\hline
\end{tabular}

The two levels of the grammar define together the class of SOA systems managed by MOSES. Given a composite service whose business logic corresponds to an instance generated by the first level rules, different combinations of the second level rules lead 
to different implementations, each characterized by different values of its overall QoS attributes. Hence, from the perspective of the structural model presented in this section, the goal of the MOSES framework is to determine at each change of the operating environment, which second level production rules should be used to bind each abstract service to a suitable implementation (and how possibly switch different requests in a flow among them, if more than one implementation is selected), to meet the QoS objectives in the new environment.

To achieve this goal, a key point is to calculate the value of the overall QoS attributes for each implementation corresponding to an instance generated by the two level grammar. In the next subsection we present the behavioral model used within MOSES for this purpose.

\subsection{Behavioral and QoS Model}

The overall QoS of a composite service implementation depends on the QoS of the concrete services that have been bound to the abstract services, on the way they are orchestrated, and on the usage profile of those services for a given class of users: a rarely invoked service has obviously a smaller impact on the overall QoS than a frequently invoked one, and different users may invoke the same services with different frequencies ${ }^{2}$.

For each user $u \in U$ (where $U$ denotes the set of the considered users), we model its usage profile by a labeled syntax tree. The syntax tree describes the "first level" production rules used to generate the composite service logical structure. Each leaf node of this tree (corresponding to an abstract service $S_{i}$ ) is labeled by $V_{i}^{u}$, the average number of times $S_{i}$ is invoked for each $u$ request addressed to the composite service. MOSES performs a monitoring activity to keep up to date the $V_{i}^{u}$ values.

Figure 3 shows the usage profile model maintained by MOSES for the composite service depicted in Figure 2.

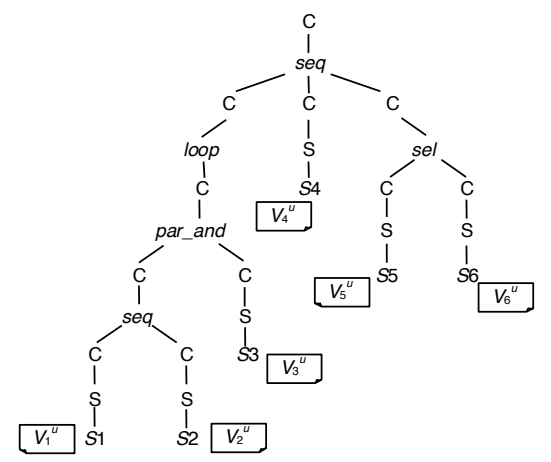

Figure 3: Usage profile model.

With this model, the $V_{i}^{u}$ values labeling the leaf nodes can be used to weigh the QoS of each $S_{i}$ implementation, while the nonterminal nodes of the syntax tree can be used to specify rules that combine these weighted QoS's to get the overall QoS.

The definition of both the QoS weight and composition rules depend on the considered QoS attributes. MOSES presently considers the following QoS attributes:

- the expected response time $R^{u}$, which is the average time needed to fulfill a user- $u$ request for the composite service;

${ }^{2}$ Users could also be grouped in homegeneous classes with the same profile, but this does not change the methodology.
- the expected execution cost $C^{u}$, which is the average price to be paid for a user- $u$ invocation of the composite service;

- the expected reliability $D^{u}$, which is the logarithm of the probability that the composite service completes its task for a user- $u$ request.

As in [27], we consider the logarithm of the reliability, rather than the reliability itself, to obtain linear expressions when composing the reliability of different services. Let us denote by $R\left(S_{i}\right)$,

Table 3: Recursive rules to calculate the average value of the QoS attributes of a composite service C, for a user $u$.

\begin{tabular}{|c|l|}
\hline $\begin{array}{c}\text { "First level" } \\
\text { node }\end{array}$ & $\begin{array}{c}\text { QoS rules } \\
\left(\text { where } \mathrm{Z}^{u}=\mathrm{C}^{u}\left|\mathrm{D}^{u}\right| \mathrm{R}^{u}\right)\end{array}$ \\
\hline $\mathrm{C}$ & $\mathrm{Z}^{u}(\mathrm{C})=\mathrm{Z}^{u}(d(\mathrm{C}))$ \\
\hline $\mathrm{S}$ & $\mathrm{Z}^{u}(\mathrm{~S})=\mathrm{Z}^{u}(d(\mathrm{~S}))$ \\
\hline $\operatorname{seq}\left(\mathrm{C}^{+}\right)$ & $\mathrm{Z}^{u}\left(\operatorname{seq}\left(\mathrm{C}^{+}\right)\right)=\sum_{c \in \mathrm{C}^{+}} \mathrm{Z}^{u}(c)$ \\
\hline loop $(\mathrm{C})$ & $\mathrm{Z}^{u}(\operatorname{loop}(\mathrm{C}))=\mathrm{Z}^{u}(\mathrm{C})$ \\
\hline sel $\left(\mathrm{C}^{+}\right)$ & $\mathrm{Z}^{u}\left(\operatorname{sel}\left(\mathrm{C}^{+}\right)\right)=\sum_{c \in \mathrm{C}^{+}} \mathrm{Z}^{u}(c)$ \\
\hline par_and $\left(\mathrm{C}^{+}\right)$ & $\mathrm{C}^{u}\left(\right.$ par_and $\left.\left(\mathrm{C}^{+}\right)\right)=\sum_{c \in \mathrm{C}^{+}} \mathrm{C}^{u}(c)$ \\
& $\begin{array}{l}\mathrm{D}^{u}\left(\text { par_and }\left(\mathrm{C}^{+}\right)\right)=\sum_{c \in \mathrm{C}^{+}} \mathrm{D}^{u}(c) \\
\mathrm{R}^{u}\left(\text { par_and }\left(\mathrm{C}^{+}\right)\right)=\max _{c \in \mathrm{C}^{+}}\left\{\mathrm{R}^{u}(c)\right\}\end{array}$ \\
\hline$S_{i}$ & $\mathrm{Z}^{u}(\mathrm{~S})=V_{i}^{u} \cdot Z\left(S_{i}\right)$ \\
\hline
\end{tabular}

$C\left(S_{i}\right)$ and $D\left(S_{i}\right)$ the average response time, cost and reliability of a given implementation of $S_{i}$. Table 3 provides rules to recursively calculate $R^{u}, C^{u}$ and $D^{u}$, given $R\left(S_{i}\right), C\left(S_{i}\right)$ and $D\left(S_{i}\right)$, $1 \leq i \leq m$. In these rules, $d(X)$ denotes the child node of a node $X$. These rules define a visit algorithm of the labeled syntax tree modeling the usage profile for a user $u \in U$, from which we get:

$$
R^{u}=\mathrm{R}^{u}(\mathrm{C}) ; \quad C^{u}=\mathrm{C}^{u}(\mathrm{C}) ; \quad D^{u}=\mathrm{D}^{u}(\mathrm{C})
$$

To complete the calculation of $R^{u}, C^{u}$ and $D^{u}$ for a given implementation of the composite service, we need to know the value of $R\left(S_{i}\right), C\left(S_{i}\right)$ and $D\left(S_{i}\right)$. These values depend on the pattern(s) used to implement $S_{i}$ and on the QoS attributes of the concrete service(s) coordinated by each pattern.

In the next section we complete this calculation and show how the model defined in this section can be used to derive the template of an optimization problem. MOSES builds and solves a new instance of this problem at each relevant change of the operating scenario, to drive the system adaptation to the new scenario.

\section{OPTIMAL ADAPTATION}

\subsection{Adaptation Policy Model}

We call an adaptation policy the set of directives issued by MOSES to select the "best" implementation of the composite service in a given scenario. We recall that MOSES assumes a flow-based service demand model with multiple concurrent users. Moreover, it can switch different requests in a flow among different patterns to implement each abstract services $S_{i}$.

Hence, determining an adaptation policy consists in determining, for each user $u$ and each abstract service $S_{i}$ :

- the coordination pattern(s) and the corresponding list of concrete services to be used to build concrete implementation(s) for $S_{i}$ (selected among the single, alt and par_or patterns).

- the fraction of requests generated by $u$ for $S_{i}$ that must be switched and bound to a specific implementation of $S_{i}$. 
To this end, we model an adaptation policy for a set $U$ of users by associating with each $u \in U$ a vector $\boldsymbol{x}^{u}=\left[\boldsymbol{x}_{1}^{u}, \ldots, \boldsymbol{x}_{m}^{u}\right]$, where $\boldsymbol{x}_{i}^{u}=\left[x_{i J}^{u}\right]$ and $0 \leq x_{i J}^{u} \leq 1$. The index $i$ of $x_{i J}^{u}$ ranges over the set of all abstract services, while the index $J$ of $x_{i J}^{u}$ ranges over the following set:

$$
J \in \Im i=\left(\mathcal{K}_{i} \cup\left(\overline{\mathcal{K}}_{i}^{+} \times\{a l t\}\right) \cup\left(\overline{\mathcal{K}}_{i}^{+} \times\{\text {par_or }\}\right)\right)
$$

In this definition $\mathcal{K}_{i}=\left\{k_{i 1}, k_{i 2}, \ldots, k_{i n_{i}}\right\}$ is the set of available concrete services implementing $S_{i}$, and $\mathcal{K}_{i}^{+}$is the set of all the ordered lists of elements of $\mathcal{K}_{i}$, where each element appears at most once (excluded the empty list) ${ }^{3}$.

For each abstract service $S_{i}, x_{i J}^{u}$ denotes the fraction of the requests generated by $u$ for $S_{i}$ to be bound to the implementation denoted by the index $J$. We have the following cases:

- $J \in \mathcal{K}_{i}$ : in this case, the index $J$ denotes a single concrete service, and $x_{i J}^{u}$ denotes the fraction of requests for $S_{i}$ to be bound to an implementation consisting of that single service;

- $J \in \overline{\mathcal{K}}_{i}^{+} \times\{$alt $\}$: in this case, the index $J$ denotes a list of concrete services coordinated according to the alt pattern, and $x_{i J}^{u}$ denotes the fraction of requests for $S_{i}$ to be bound to an implementation consisting of those services;

- $J \in \overline{\mathcal{K}}_{i}^{+} \times\{$par_or $\}$: in this case, the index $J$ denotes a list of concrete services coordinated according to the par_or pattern, and $x_{i J}^{u}$ denotes the fraction of requests for $S_{i}$ to be bound to an implementation consisting of those services.

As an example, consider the case $\mathcal{K}_{i}=\left\{k_{i 1}, k_{i 2}, k_{i 3}\right\}$ and assume that the adaptation policy $\boldsymbol{x}_{i}^{u}$ for a given user $u$ specifies the following values: $x_{i\left\{k_{i 1}\right\}}^{u}=x_{i\left\{k_{i 3}\right\}}^{u}=0.3, x_{i\left\{k_{i 2}, k_{i 3}\right\} \times \text { par_or }}^{u}=$ 0.4 and $x_{i J}^{u}=0$ otherwise. This strategy implies that $30 \%$ of the user $u$ requests for service $S_{i}$ are bound to service $k_{i 1}, 30 \%$ are bound to service $k_{i 3}$ while the remaining $40 \%$ are bound to the pair $k_{i 1}, k_{i 3}$ coordinated by the par_or pattern (see Figure 4 ). From this example we can see that, to get some overall QoS objective for the flow generated by a user, MOSES may switch different requests in this flow to different implementations (using $\boldsymbol{x}_{i}^{u}$ to drive the switch). Moreover, it may select implementations that use in different ways the same concrete service ( $k_{i 3}$ in this example).

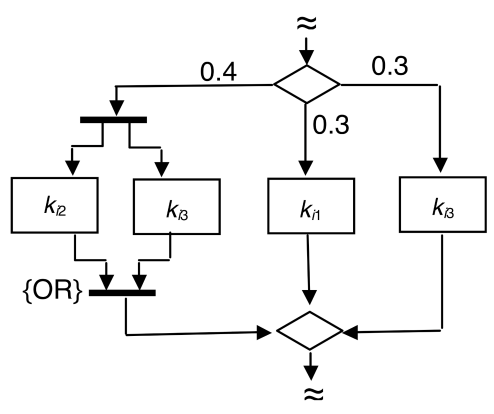

Figure 4: Flow partitioning among different implementations.

\footnotetext{
${ }^{3}$ We point out that not all the instances generated by the second level production rules of Section 3 correspond to meaningful implementations (for example, those where a concrete service $k_{i j}$ is repeated more than once in the par_or pattern). $\overline{\mathcal{K}}_{i}^{+}$considers a meaningful subset of such instances.
}

\subsection{Policy-based QoS Metrics}

Given an adaptation policy $\boldsymbol{x}$, we calculate in this section the QoS attributes $R^{u}(x), C^{u}(x)$ and $D^{u}(x)$ experienced by a user $u \in U$ under that policy.

To this end, we denote by $\left\langle r_{i j}, c_{i j}, d_{i j}, L_{i j}\right\rangle$ the parameters of the SLA negotiated with the provider of the concrete service $k_{i j}$ that implements the abstract service $S_{i}\left(1 \leq i \leq m, 1 \leq j \leq n_{i}\right)$, where $r_{i j}, c_{i j}$, and $d_{i j}$ are the average response time, cost, and logarithm of reliability of $k_{i j}$. As we are in a flow-based setting, the SLA states that these values hold on the average for all the requests in a flow, and are guaranteed as long as the rate of requests to $k_{i j}$ does not exceed the $L_{i j}$ threshold.

Given the SLAs parameters, we can determine the value of the QoS attributes $R\left(S_{i}\right), C\left(S_{i}\right)$ and $D\left(S_{i}\right)$. Let us denote by $R\left(S_{i} ; J\right)$, $C\left(S_{i} ; J\right)$ and $D\left(S_{i} ; J\right)$ the average response time, cost and logarithm of reliability of $S_{i}$, when $S_{i}$ is implemented according to the pattern denoted by an index $J \in \Im i$. We distinguish among the three patterns:

- $J \in \mathcal{K}_{i}$ : assuming $\mathcal{K}_{i}=\left\{k_{i j}\right\}$, the QoS attributes coincide with those of the selected concrete service $k_{i j}$ :

$$
C\left(S_{i} ; J\right)=c_{i j}, \quad D\left(S_{i} ; J\right)=d_{i j}, \quad R\left(S_{i} ; J\right)=r_{i j}
$$

- $J \in \overline{\mathcal{K}}_{i}^{+} \times\{a l t\}$ : assuming $\overline{\mathcal{K}}_{i}^{+}=\left\{k_{i j_{1}}, \ldots, k_{i j_{l}}\right\}$, the concrete services listed in $\overline{\mathcal{K}}_{i}^{+}$are tried in sequence, starting from the first in the list, until one of them successfully completes. Hence, the logarithm of reliability of this pattern is derived from the probability that at least one service completes, while the cost and time to completion of all the elements of the list must be summed, each weighed by the probability that the invocations of all the preceding elements in the list have failed (where, for the sake of readability, we use $a_{i j}=e^{d_{i j}}$, i.e., $a_{i j}$ is the reliability of $k_{i j}$ ):

$$
\begin{aligned}
& C\left(S_{i} ; J\right)=\sum_{h=1}^{l} c_{i j_{h}} a_{i j_{h}} \prod_{s=1}^{h-1}\left(1-a_{i j_{s}}\right) \\
& D\left(S_{i} ; J\right)=\log \left(1-\prod_{s=1}^{l}\left(1-a_{i j_{s}}\right)\right) \\
& R\left(S_{i} ; J\right)=\sum_{h=1}^{l} r_{i j_{h}} a_{i j_{h}} \prod_{s=1}^{h-1}\left(1-a_{i j_{s}}\right) N^{-1}
\end{aligned}
$$

- $J \in \overline{\mathcal{K}}_{i}^{+} \times\{$par_or $\}$: in this case, the costs of all the services in $\overline{\mathcal{K}}_{i}^{+}$must be summed as they are invoked in parallel:

$$
\begin{aligned}
& C\left(S_{i} ; J\right)=\sum_{h=1}^{l} c_{i j_{h}} \\
& D\left(S_{i} ; J\right)=\log \left(1-\prod_{s=1}^{l}\left(1-a_{i j_{s}}\right)\right) \\
& R\left(S_{i} ; J\right)=\sum_{K \in 2^{J} \backslash\{\oslash\}}\left(\prod_{j \in K} a_{i j} \prod_{j \in J \backslash K}\left(1-a_{i j}\right)\right) \min _{j \in K}\left\{r_{i j}\right\} N^{-1}
\end{aligned}
$$

In the expressions for $R\left(S_{i} ; J\right), N=\left(1-\prod_{h=1}^{l}\left(1-a_{i j_{h}}\right)\right)$ is a normalization factor that accounts for the fact that at least one service terminates. We point out that the expression for $R\left(S_{i} ; J\right)$ in (3) is exact under the assumption that the average value of the minimum of the response times of the services in $\overline{\mathcal{K}}_{i}^{+}$is equal to the minimum of their average values. This assumption holds when the distribution of the response times is deterministic, otherwise the expression represents an approximation, which is quite accurate for distributions with low variance. In other cases a more suitable 
expression should be used, which would require the knowledge of the response time distribution, but this is out of the scope of this paper.

From (1), (2) and (3), we see that the implementations of $S_{i}$ according to the alt or par_or patterns have the same reliability when they use the same set of services. This reliability is higher than the reliability of the single pattern (for any single service belonging to the set used by alt or par_or). On the other hand, it is easy to verify (with some algebra) that alt has a lower cost than par_or, but a higher response time, since the sequential invocation used by alt means that on the average not all the selected services are invoked, but the response time of those invoked must be summed. Moreover, under the "deterministic" assumption discussed above, the par_or pattern does not improve the response time with respect to the best selection of a service under the single pattern, but has a higher cost. par_or would improve the response time over single in case of distribution of the response time with non zero variance (e.g., exponential).

Given expressions (1), (2) and (3) for $C\left(S_{i} ; J\right), D\left(S_{i} ; J\right)$ and $R\left(S_{i} ; J\right)$, we can complete the calculation of $C^{u}(\boldsymbol{x}), D^{u}(\boldsymbol{x})$ and $R^{u}(\boldsymbol{x})$. From the recursive rules of Table 3 , we can easily derive the following closed forms for $C^{u}(\boldsymbol{x})$ and $D^{u}(\boldsymbol{x})$ :

$$
\begin{aligned}
& C(\boldsymbol{x})=\sum_{i=1}^{m} V_{i}^{u} \sum_{J \in \Im i} x_{i J}^{u} C\left(S_{i} ; J\right) \\
& D(\boldsymbol{x})=\sum_{i=1}^{m} V_{i}^{u} \sum_{J \in \Im i} x_{i J}^{u} D\left(S_{i} ; J\right)
\end{aligned}
$$

For $R^{u}(\boldsymbol{x})$, we need to account for the fact that the overall response time of the par_and pattern is the largest response time among its component activities. In this case, the response time is no longer additive and we cannot derive an expression analogous to (4) and (5). However, we have shown in [7] that we can still derive the following recursive expression for $R^{u}(\boldsymbol{x})$ using the labeled syntax tree (we omit the proof here for space reasons):

$$
\begin{aligned}
& R^{u}(\boldsymbol{x})=R_{\text {root }}^{u}(\boldsymbol{x}) \\
& R_{l}^{u}(\boldsymbol{x})= \begin{cases}\max _{l^{\prime} \in d(l)} R_{l^{\prime}}^{u}(\boldsymbol{x}) & l \in \Pi \\
\sum_{i \prec_{d d} l} \frac{V_{i}^{u}}{V_{l}^{u}} \sum_{J \in \Im i} x_{i J}^{u} R\left(S_{i} ; J\right)+ & \\
+\sum_{h \in \Pi, h \prec_{d d} l} \frac{V_{h}^{u}}{V_{l}^{u}} R_{h}^{u}(\boldsymbol{x}) & l \notin \Pi\end{cases}
\end{aligned}
$$

In (6), root denotes the root node of the labeled syntax tree, $l$ is a generic node of the tree, $\Pi$ denotes the set of par_and nodes in the tree, and $\prec_{d d}$ is an order relationship among the nodes of the tree: given two nodes $i$ and $h, h \prec_{d d} i$ basically means that node $h$ belongs to the sub-tree rooted at $i$ and, within this sub-tree, $h$ does not appear within a par_and activity (see [7] for details). We use this general closed form in the formulation of the optimization problem reported below.

\subsection{Optimization Problem}

We can now formulate the optimization problem solved by MOSES to determine the optimal policy $\boldsymbol{x}$ in a given environment. To this end, we denote by $\left\langle R_{\max }^{u}, C^{u}, D_{\min }^{u}, L^{u}\right\rangle$ the parameters of the SLA negotiated by a user $u$ for the access to the composite service managed by MOSES. $R_{\max }^{u}$ is an upper bound on the average response time the user $u$ is willing to experience, while $D_{\min }^{u}$ is a lower bound on the logarithm of the reliability. These bounds hold as long as the rate of service requests generated by $u$ does not exceed the threshold $L^{u}$. $C^{u}$ is the cost $u$ pays for each invocation of the composite service.

We assume that MOSES wants, in general, to define an adaptation policy that optimizes multiple - possibly conflicting - re- quirements, within the constraints defined by the environment. We tackle this multi-objective problem by transforming it into a single objective problem, where the objective function $F(\boldsymbol{x})$ is an aggregate QoS measure given by the weighted sum of the (normalized) QoS attributes of all users. More precisely, let $Z(x)=$ $\frac{\sum_{u \in U} L^{u} Z^{u}(\boldsymbol{x})}{\sum_{u \in U} L^{u}}$ where $Z=R|D| C$ is the expected overall response time, reliability and cost, respectively. We define the objective function as follows:

$F(\boldsymbol{x})=w_{r} \frac{R_{\max }-R(\boldsymbol{x})}{R_{\max }-R_{\min }}+w_{d} \frac{D(\boldsymbol{x})-D_{\min }}{D_{\max }-D_{\min }}+w_{c} \frac{C_{\max }-C(\boldsymbol{x})}{C_{\max }-C_{\min }}$

where $w_{r}, w_{d}, w_{c} \geq 0, w_{r}+w_{d}+w_{c}=1$, are weights for the different QoS attributes. $R_{\max }\left(R_{\min }\right), D_{\max }\left(D_{\min }\right)$, and $C_{\max }$ $\left(C_{\mathrm{min}}\right)$ denote, respectively, the maximum (minimum) value for the overall expected users response time, cost and the (logarithm of) reliability. We will describe how to determine these values shortly. $F(\boldsymbol{x})$ takes values in the interval $[0,1]$.

With these definitions, the optimization problem can be formulated as follows:

$$
\begin{gathered}
\max F(\boldsymbol{x}) \\
\text { subject to: } C^{u}(\boldsymbol{x}) \leq C^{u}, \quad u \in U \\
D^{u}(\boldsymbol{x}) \geq D_{\min }^{u}, \quad u \in U \\
R^{u}(\boldsymbol{x}) \leq R_{\max }^{u}, \quad u \in U \\
R_{l^{\prime}}^{u}(\boldsymbol{x}) \leq R_{l}^{u}(\boldsymbol{x}), \quad l^{\prime} \in d(l), l \in \Pi, u \in U \\
R_{l}^{u}(\boldsymbol{x})=\sum_{i \prec d d} \frac{V_{i}^{k}}{V_{l}^{k}} \sum_{J \in \Im i} x_{i J}^{u} R\left(S_{i} ; J\right)+ \\
+\sum_{h \in \Pi, h \prec d d} \frac{V_{h}^{u}}{V_{l}^{u}} R_{h}^{u}(\boldsymbol{x}), l \notin \Pi, u \in U \\
\sum_{u \in U} \sum_{J \in \Im i, j \in J} x_{i J}^{u} V_{i}^{u} L^{u} \leq L_{i j}, \quad 1 \leq i \leq m, 1 \leq j \leq n_{i} \\
x_{i J}^{u} \geq 0, J \in \Im i, \sum_{J \in \Im i} x_{i J}^{u}=1, \quad 1 \leq i \leq m, u \in U
\end{gathered}
$$

Equations (8)-(12) are the QoS constraints for each user on the cost, reliability and response time, where $C^{u}, D_{\min }^{u}$, and $R_{\max }^{u}$ are the SLA parameters negotiated between the system managed by MOSES and user $u$. The constraints (10)-(12) for the response time take into account the peculiarity in the estimation of this attribute. Inequalities (11), in particular, allow us expressing the relationship among the response time $R_{l}^{u}(\boldsymbol{x})$ of a par_and activity and that of its component activities $R_{l^{\prime}}^{u}(\boldsymbol{x})$, where for each par_and activity $l$ we denote by $d(l)$ the set of top-level activities/services which are nested within $l$ in the syntax tree.

Equations (13) are the SLA constraints negotiated with the providers of the component services, and ensure that the system managed by MOSES does not exceed the volume of invocations agreed with those providers. Finally, Equations (14) are the functional constraints.

The maximum and minimum values of the QoS attributes in the objective function (7), used to get a normalized value, are determined as follows. $R_{\max }, C_{\max }$, and $D_{\min }$ are simply expressed respectively in terms of $R_{\max }^{u}, C^{u}$, and $D_{\max }^{u}$. For example, the maximum response time is given by $R_{\max }=\frac{\sum_{u \in U} L^{u} R_{\max }^{u}}{\sum_{u \in U} L^{u}}$. Similar expressions hold for $C_{\max }$ and $D_{\min }$. The values for $R_{\min }$, $C_{\min }$, and $D_{\max }$ are determined by solving a modified optimization problem in which the objective function is the QoS attribute of interest, subject to the constraints (13)-(14).

We observe that the proposed optimization problem is a Linear Programming problem which can be efficiently solved via standard techniques. This efficiency could be outweighed by the high num- 
ber of variables $x_{i J}^{u}$ in the optimization problem, which is exponential in the number of concrete services implementing each abstract service. A possible way to tackle this problem is to restrict the number of considered subsets $J$ to those having at most a given cardinality, considering the diminishing QoS increase we can achieve with higher redundancy levels.

\section{MOSES IMPLEMENTATION AND NUMERICAL EXPERIMENTS}

In this section we describe the prototype tool that implements the MOSES methodology and discuss numerical experiments that illustrate the kind of adaptation directives issued by MOSES. These directives are determined as solution of the optimization problem described in the previous section.

\subsection{MOSES Prototype}

This section describes our ongoing prototype implementation of MOSES. As shown in Figure 5, the prototype consists of three main layers (Implementation, Runtime Monitoring, and Adaptation) and three auxiliary components (Workflow Validation, Model Generation, and Service Discovery \& SLA Negotiation). The Implementa-

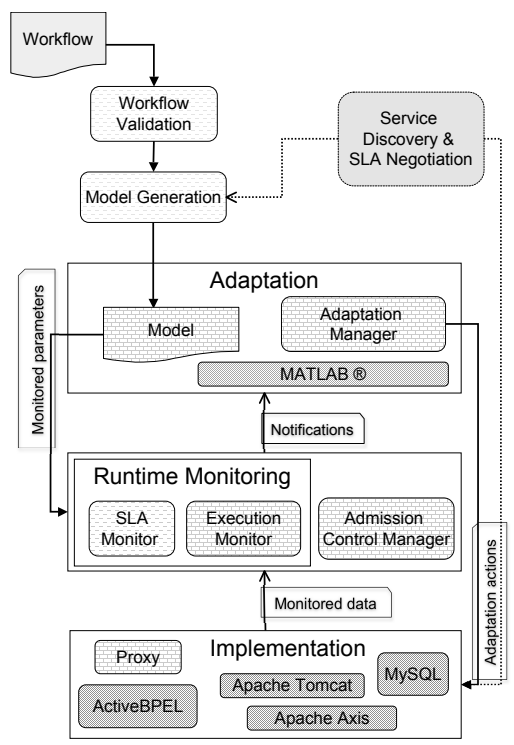

Figure 5: The MOSES prototype architecture (dark grey boxes indicate the supporting technologies).

tion layer is the software platform that executes the business process with the opportune adaptation actions driven by the Adaptation layer and represents the user front-end for the composite service provisioning. This layer is responsible for managing the user requests flow, once the user has been admitted to the system with an established SLA. It has been developed using open source products: ActiveBPEL for the BPEL engine, Apache Axis and Apache Tomcat for the SOAP engine and servlet container respectively, and MySQL for the repository. The latter stores all the information needed by MOSES to carry out the runtime adaptation (among which the abstract and the corresponding concrete services with their QoS values, and the $x_{i J}^{u}$ values determined by the solution of the optimization problem). When a user invokes the business process, ActiveBPEL creates a new instance of the process itself. Each generated instance can differ according to the solution of the optimization problem determined by the Adaptation layer and stored in the repository. As already discussed, for a given abstract service the requests of users with different (or even the same) SLAs can be bound to different concrete services, also using different coordination patterns.

The current MOSES prototype supports the three coordination patterns listed in Table 2 by means of the Proxy component, similarly to the proxy-based approach in [12] which addresses reliability support in a BPEL process. The Implementation layer uses the solution $x$ of the optimization problem to drive the Proxy that selects, for each invocation of an abstract service $S_{i}$, the specific implementation to which it must be bound. The Proxy, written in Java, has been designed to support the coordination patterns in a transparent way to the BPEL engine and the BPEL process (apart from some patches that are automatically applied by MOSES to the BPEL process in order to let the process call the Proxy rather than other endpoints). While the single pattern is simply implemented by selecting the concrete service identified by the solution of the optimization problem, the alt pattern requires sequential invoke operations within a loop on the list of alternate concrete services determined at runtime by the solution of the optimization problem. Finally, the par_or pattern (which does not have a corresponding BPEL construct) has been implemented by means of the flow, throw, and catch activities.

The Runtime Monitoring layer consists of the Execution Monitor and SLA Monitor modules. The Execution Monitor collects and keeps up to date information about the composite service usage profile, calculating estimates of the $V_{i}^{u}$ values. The SLA Monitor collects information about the performance and reliability levels (specified in the SLAs) perceived by the users and offered by the providers of the component services, and about the mean volume of requests generated by the users. Furthermore, the SLA Monitor gives warning to the Adaptation layer whether there is some variation in the pool of concrete services available for a given abstract service. Our current implementation of the SLA Monitor measures the mean volume of requests generated by the users, as well as the response time and reliability offered by the providers to MOSES. To monitor other SLA parameters and estimate the network impact on the user performance, the prototype could also rely on third party SLA monitors, such as those provided by Keynote Systems [18]. The Admission Control Manager does not play a true monitoring role, but rather manages new user arrivals to find out whether a new user can be accepted by the system, given its QoS expectations and the already existing SLAs. Moreover, it notifies to the Adaptation Manager the fluctuation of workload intensity parameters caused by the arrival or departure of users.

In the Adaptation layer, we have the business process model and the Adaptation Manager. The business process model defines the parameters that will be observed by the Runtime Monitoring layer during the business process execution. Upon receiving a notification of a significant variation in the model parameters (from the latter layer), the Adaptation Manager finds out whether and how an adaptation action has to be performed. To this end, it solves the optimization problem described in Section 4.3, using the new instance of the system model with the changed values of the parameters. The calculated solution provides indications about the adaptation actions that must be performed to optimize the use of the available resources (i.e., the concrete services) with respect to MOSES optimization criterion. Based on this solution, the Adaptation Manager issues suitable directives to the Implementation layer, so that future instances of the business process will be served according to these directives. The construction and solution of the optimization problem is implemented in MATLAB.

The auxiliary components, only partially implemented in our 
prototype, perform the following tasks. The Workflow Validation parses the BPEL workflow and verifies whether its structure is compliant with MOSES, as specified in Section 3.1. In the positive case, the Model Generation produces from the workflow specified in BPEL code the behavioral model of the composite service. Finally, the Service Discovery \& SLA negotiation is responsible to find the service providers offering functionally equivalent service implementations and to negotiate with them the SLAs. This latter component, although necessary for the system usage at production time, is currently out of the scope of our research.

\subsection{Numerical Experiments}

In this section, we illustrate the behavior of the proposed adaptation strategy scheme through the simple abstract workflow of Figure 2. For the sake of simplicity we assume that two candidate concrete services (with their respective SLAs) have been identified for each abstract service except for service $S_{2}$ for which four concrete services have been identified. The respective SLAs differ in terms of cost, reliability and response time. Table 4 summa-

Table 4: Concrete services SLA parameters.

\begin{tabular}{|c|c|c|c|c|c|c|c|}
\hline Serv. & $c_{i j}$ & $d_{i j}$ & $r_{i j}$ & Serv. & $c_{i j}$ & $d_{i j}$ & $r_{i j}$ \\
\hline \hline$k_{11}$ & 6 & $\log (0.995)$ & 2 & $k_{32}$ & 1.8 & $\log (0.95)$ & 2 \\
\hline$k_{12}$ & 3 & $\log (0.99)$ & 4 & $k_{41}$ & 1 & $\log (0.995)$ & 0.5 \\
\hline \hline$k_{21}$ & 4 & $\log (0.99)$ & 2 & $k_{42}$ & 0.8 & $\log (0.99)$ & 1 \\
\hline$k_{22}$ & 2 & $\log (0.95)$ & 4 & $k_{51}$ & 2 & $\log (0.99)$ & 2 \\
\hline$k_{23}$ & 4.5 & $\log (0.99)$ & 1 & $k_{52}$ & 1.4 & $\log (0.95)$ & 4 \\
\hline$k_{24}$ & 1 & $\log (0.95)$ & 4 & $k_{61}$ & 0.5 & $\log (0.99)$ & 1.8 \\
\hline \hline$k_{31}$ & 2 & $\log (0.995)$ & 1 & $k_{62}$ & 0.4 & $\log (0.9)$ & 4 \\
\hline
\end{tabular}

rizes the SLA parameters $\left\langle r_{i j}, c_{i j}, d_{i j}\right\rangle$ for each concrete service $k_{i j}$. They have been chosen so that for abstract service $S_{i}$, concrete service $k_{i 1}$ represents the better service, which at a higher cost guarantees higher reliability and lower response time with respect to service $k_{i 2}$, which costs less but has lower reliability and higher response time. For all services, $L_{i j}=10$. On the user side,

Table 5: User SLA parameters.

\begin{tabular}{|c|c|c|c|c|}
\hline User & $C^{u}$ & $D_{\min }^{u}$ & $R_{\max }^{u}$ & $L^{u}$ \\
\hline \hline 1 & 25 & $\log (0.99)$ & 7 & 1.5 \\
\hline 2 & 18 & $\log (0.95)$ & 11 & 1 \\
\hline 3 & 15 & $\log (0.95)$ & 15 & 3 \\
\hline 4 & 12 & $\log (0.9)$ & 18 & 1 \\
\hline
\end{tabular}

we assume a scenario with four concurrent users of the composite service managed by MOSES. The SLAs negotiated by these users are characterized by a wide range of QoS requirements as listed in Table 5, with User 1 having the most stringent requirements, $D_{\min }^{1}=\log (0.99)$ and $R_{\max }^{1}=7$ and User 4 the least stringent requirements $D_{\text {min }}^{4}=\log (0.9)$ and $R_{\max }^{4}=18$. The SLA costs parameters for these users have been set accordingly, where User 1 has accepted to pay the highest cost per request, $C^{1}=25$, while User 4 only $C^{4}=12$. The usage profile of the different users is given by the following values for the expected number of service invocations: $V_{1}^{u}=V_{2}^{u}=V_{3}^{u}=1.5, V_{4}^{u}=1, u \in U ; V_{5}^{u}=0.7$, $V_{6}^{u}=0.3, u \in\{1,3,4\} ; V_{5}^{2}=V_{6}^{2}=0.5$. In other words, all users have the same usage profile except for User 2, who invokes the services 5 and 6 with different intensity.

We illustrate the adaptation directives issued by MOSES assuming that its optimization goal is the minimization of the average cost to implement the composite service, i.e., $w_{c}=1$ (this goal could corresponds to the income maximization for the composite service provider). The results are summarized in Figures 6(a) and 6(b) that show the adaptation policies for User 1 and User 4, and in Table 6 where we list the resulting QoS metrics for all the users. Observe that under cost minimization, there is no incentive to guarantee to the users more than the minimum required. Thus, the solution calculated by MOSES guarantees only the minimum required level of reliability and response time, i.e., $D^{u}(x)=D_{\min }^{u}$ and $R^{u}(x)=R_{\max }^{u}$ (see Tables 5 and 6), with increasing costs for increasing QoS user requirements.

Table 6: QoS metrics achieved by MOSES.

\begin{tabular}{|c|c|c|c|}
\hline User & $C^{u}(\boldsymbol{x})$ & $D^{u}(\boldsymbol{x})$ & $R^{u}(\boldsymbol{x})$ \\
\hline \hline 1 & 21.18 & $\log (0.99)$ & 7 \\
\hline 2 & 15.27 & $\log (0.95)$ & 11 \\
\hline 3 & 12.32 & $\log (0.95)$ & 15 \\
\hline 4 & 10.71 & $\log (0.9)$ & 18 \\
\hline
\end{tabular}

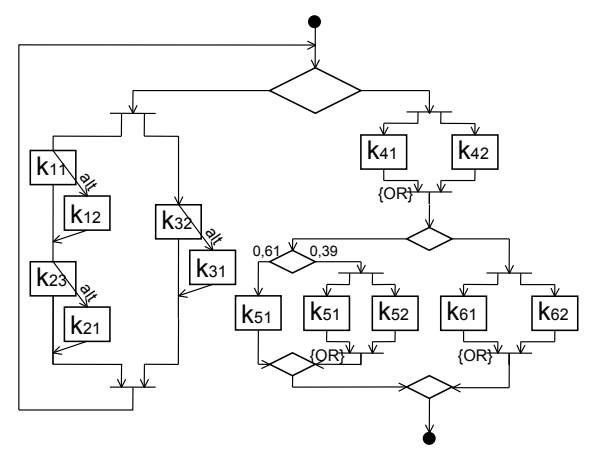

(a) User 1

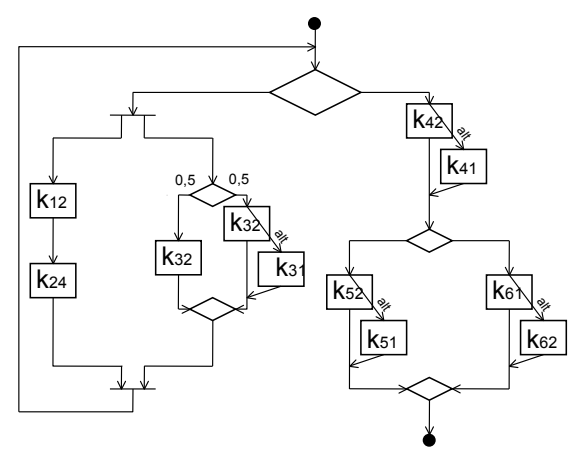

(b) User 4

\section{Figure 6: Adaptation policies for the workflow of Figure 2.}

The resulting workflow for User 4 employs only the single and the alt coordination patterns (see Figure 6(b)): services $S_{1}$ and $S_{2}$ are bound to the cheaper $k_{12}$ and $k_{24}$, respectively; for the other services, the solution bounds them to an implementation based on the alt pattern which, with the exception of $S_{6}$, takes the form of $\operatorname{alt}\left(k_{i 2}, k_{i 1}\right)$ (observe that $\operatorname{alt}\left(k_{i 2}, k_{i 1}\right)$ and $\operatorname{alt}\left(k_{i 1}, k_{i 2}\right)$ have the same reliability, but with our choice of concrete service QoS parameters, the former pattern is always characterized by a lower cost and higher response time). In the case of $S_{3}$, note that a fraction of the traffic is switched to an implementation based on the alt pattern. 
The solution for User 1, who has more stringent QoS requirements, differs substantially from the one just described. From the figure we observe that User 1 workflow employs a redundant solution for each service, and adopts the par_or pattern to implement some services (remember that the par_or pattern for the same level of reliability of the alt pattern yields lower response time at a higher cost), which comes at a significant higher cost per request (21.18, almost twice the 10.71 needed to satisfy a User 4 request).

To illustrate how the optimal adaptation changes upon environment variations, we now assume that the monitoring service notifies MOSES that service $k_{32}$ is not longer available. In this case, MOSES builds and solves a new instance of the optimization problem. The new solution has the same level of reliability and response time but higher cost for all users (we do not show the actual values for space limitation). For User 1 we show the new adaptation policy in Figure 7. We can observe that in the new optimal solution the decrease of reliability of the implementation of $S_{3}$ due to the disappearance of concrete service $k_{32}$ is compensated by an increase of reliability of $S_{5}$ which is now implemented only by par_or $\left(k_{51}, k_{52}\right)$. Moreover in the implementation of $S_{4}$, $\operatorname{alt}\left(k_{41}, k_{42}\right)$, which has the same level of reliability but costs less, replaces par_or $\left(k_{41}, k_{42}\right)$.

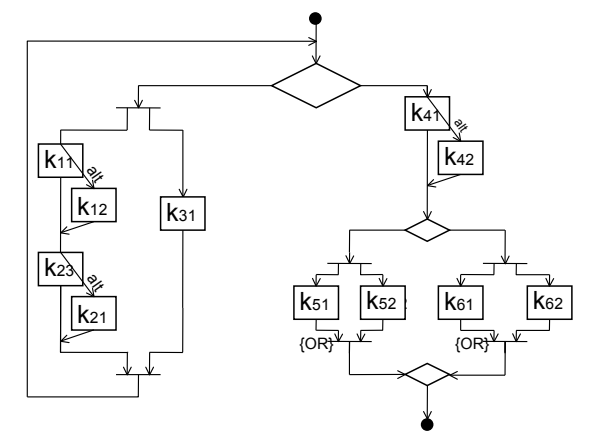

Figure 7: New adaptation policy for User 1.

\section{RELATED WORK}

As outlined in [10], the topic of self-adaptive computing systems has been studied in several communities and from different perspectives. The autonomic computing framework is a notable example of general approach to the design of such systems [16]. As indicated in the original proposal by IBM [17], the architecture of an autonomic system consists of a set of managers and managed resources. The manager communicates with the resource through a sensor/actuator mechanism and the decision is elaborated using the so-called MAPE-K (Monitor, Analyze, Plan, Execute and Knowledge) cycle. This loop collects information from the system, makes decisions and then organizes the actions needed to achieve goals and objectives, and controls the execution. We have outlined in Section 3 the relationship between MOSES and the MAPE cycle. Hereafter, we focus on works appeared in the literature dealing with issues concerning the QoS evaluation and the self-adaptation of SOA systems, to guarantee the fulfillment of QoS requirements.

A basic problem to be solved when dealing with QoS issues of SOA systems, is how to determine the QoS attributes of a composite system, given the QoS delivered by its component services. Some papers have focused on this specific issue [8, 19, 24], while others deal with it as a step within the more general problem of QoS based model-driven runtime adaptation of SOA systems [2,
$1,3,5,7,9,13,14,23,25,26,27]$. Some of the works dealing with this general problem propose heuristics (e.g., [3, 13] or genetic algorithms in [5]) to determine the adaptation actions. Others propose exact algorithms to this end: Yu and Lin [26] formulate a multi-dimension multi-choice 0-1 knapsack problem as well as a multi-constraint optimal path problem; Zeng et al. [27] present a global planning approach to select an optimal execution plan by means of integer programming; in [2, 13, 23] the adaptation actions are selected through mixed integer programming.

As already outlined in the introduction, with regard to the mechanisms used to carry out the adaptation, most of these papers have focused on dynamic service selection. Other papers have instead considered workflow restructuring, exploiting the inherent redundancy of the SOA environment $[9,13,14,25]$. [13] provides a methodology to select different redundancy mechanisms to improve the reliability experienced by a single request addressed to a composite service. In $[9,14,25]$ the SOA environment redundancy is used as a way to adapt workflow by identifying multiple diverse workflows that achieve the same goal. In this respect, the MOSES aim is to provide a unified framework where service selection is integrated with other kinds of workflow restructuring, to achieve a greater flexibility in the adaptation of a SOA system.

An important aspect in model-driven adaptation of SOA systems concerns the assumptions underlying the proposed methodologies. In this respect, even if not always explicitly stated, proposed approaches share a common set of assumptions. They include synchronous invocation of services and stateless services. The former assumption is relevant for the estimation of the overall response time as (possibly weighted) sum of the response time of the invoked services. The latter provides the ground to freely (re-)bind different functionally equivalent services to an abstract service, and to coordinate them by redundancy patterns.

A relaxation of the stateless assumption can be found in [2], where the proposed model allows to specify that some concrete services implementing different functionalities must be bound to corresponding abstract services with an "all or none" logic.

At present, MOSES too is based on the above assumptions. On the other hand, a significant difference of MOSES from other methodologies for runtime SOA systems adaptation concerns the considered adaptation scenario. Most of the proposed approaches focus on a scenario concerning a single request addressed to a composite SOA system $[2,5,9,13,14,25,26,27]$. Their aim is to determine the adaptation action that is (possibly) optimal for that single request, independently of other concurrent requests, considering a given set of QoS requirements and the current conditions of the operating environment. MOSES instead is intended to operate in a scenario where a quite sustained traffic of requests is addressed to a SOA system. Hence, its aim is to determine adaptation actions spanning the overall flow of requests, rather than a single request. A potential drawback of this approach with respect to those focusing on single requests is that we loose the possibility of customizing the adaptation action for each request. However, in the scenario we consider, performing a per-request rather than a per-flow adaptation could cause an excessive computational load (in this respect, per-request approaches often lead to formulations of the problem to be solved as a NP-hard problem, which could thus result too complex for runtime decisions). Moreover, per-request adaptation in a sustained traffic scenario could incur in stability and management problems, since the "local" adaptation actions could conflict with adaptation actions independently determined for other concurrent requests. Preliminary versions of the per-flow approach adopted within MOSES have been presented in $[1,6,7]$. However, those papers consider only service selection as adaptation mechanism. 


\section{CONCLUSIONS}

In this paper we presented the MOSES framework for runtime QoS-driven adaptation of SOA systems. The basic guideline we have followed in its definition has been to devise an adaptation methodology that is flexible, to cope with QoS requirements that may come from different classes of users, and efficient, to make realistic its use in taking runtime decisions. We achieve flexibility by integrating within our framework different adaptation mechanisms (service selection and a kind of workflow restructuring) that can be simultaneously used to serve the requests of different users, or even different requests from the same user. We achieve efficiency by formulating the identification of the most suitable adaptation action at each detected environment change as a linear programming problem, that can be solved by using standard techniques and tools.

The methodology proposed in this paper can be extended along several directions that, besides relaxing the assumptions discussed in Section 6, include: the kind of provided guarantees on the QoS attributes, the considered coordination patterns among functionally equivalent services, and the kind of workflow structure used for the business logic of a composite service. With regard to the QoS guarantees, MOSES presently considers the average value of the QoS attributes. More general types of statistical guarantees could include, for example, an upper bound on the 99-percentile of a QoS attribute. With regard to the adopted coordination patterns for reliability improvement, we basically assume a fail-stop failure model: other failure models, e.g., Byzantine failures, would require different kinds of coordination patterns. Finally, MOSES currently manages composite services whose orchestration pattern matches the first level of the grammar presented in Section 2. We plan to include in MOSES additional orchestration patterns. Besides working on these methodological extensions, we are also working on the completion of our prototype, to validate the methodology through a thorough set of real experiments.

\section{ACKNOWLEDGEMENTS}

Work partially supported by the Italian PRIN project D-ASAP and by the project Q-ImPrESS (215013) funded under the European Union's Seventh Framework Programme (FP7).

\section{REFERENCES}

[1] D. Ardagna, C. Ghezzi, and R. Mirandola. Model driven qos analyses of composed web services. In ServiceWave, volume 5377 of $L N C S$, pages 299-311, 2008.

[2] D. Ardagna and B. Pernici. Adaptive service composition in flexible processes. IEEE Trans. Softw. Eng., 33(6):369-384, June 2007.

[3] R. Berbner, M. Spahn, N. Repp, O. Heckmann, and R. Steinmetz. Heuristics for qos-aware web service composition. In IEEE Services Computing Workshops, pages 72-82, 2006.

[4] E. Borger. Modeling workflow patterns from first principles. In ER 2007, volume 4801 of $L N C S$, pages 1-20, 2007.

[5] G. Canfora, M. D. Penta, R. Esposito, and M. L. Villani. A framework for qos-aware binding and re-binding of composite web services. J. of Systems and Software, 81(10):1754-1769, 2008.

[6] V. Cardellini, E. Casalicchio, V. Grassi, and R. Mirandola. A framework for optimal service selection in broker-based architectures with multiple QoS classes. In IEEE Services Computing Workshops, pages 105-112, 2006.

[7] V. Cardellini, E. Casalicchio, V. Grassi, and F. L. Presti. Flow-based service selection for web service composition supporting multiple qos classes. In IEEE ICWS, pages 743-750, 2007.

[8] J. Cardoso, A. P. Sheth, J. A. Miller, J. Arnold, and K. J. Kochut. Modeling quality of service for workflows and web service processes. Web Semantics J., 1(3):281-308, 2004.

[9] G. Chafle, P. Doshi, J. Harney, S. Mittal, and B. Srivastava. Improved adaptation of web service compositions using value of changed information. In IEEE ICWS, pages 784-791, 2007.

[10] B. H. C. Cheng, H. Giese, P. Inverardi, J. Magee, and R. de Lemos. 08031 - software engineering for self-adaptive systems: A research road map. In Software Engineering for Self-Adaptive Systems, volume 08031 of Dagstuhl Seminar Proceedings, 2008.

[11] H. R. D.A. Menascé and H. Gomma. Qos management in service oriented architectures. Performance Evaluation J., 7-8(64), Aug. 2007.

[12] O. Ezenwoye and S. M. Sadjadi. A language-based approach to addressing reliability in composite web services. In SEKE, pages 649-654, 2008.

[13] H. Guo, J. Huai, H. Li, T. Deng, Y. Li, and Z. Du. Angel: Optimal configuration for high available service composition. In IEEE ICWS, pages 280-287, 2007.

[14] J. Harney and P. Doshi. Speeding up adaptation of web service compositions using expiration times. In $W W W$, pages 1023-1032. ACM, 2007.

[15] Q. He, J. Yan, H. Jin, and Y. Yang. Adaptation of web service composition based on workflow patterns. In ICSOC, pages 22-37. Springer-Verlag, 2008.

[16] M. C. Huebscher and J. A. McCann. A survey of autonomic computing - degrees, models, and applications. ACM Comput. Surv., 40(3), 2008.

[17] J. O. Kephart and D. M. Chess. The vision of autonomic computing. IEEE Computer, 36(1):41-50, 2003.

[18] Keynote system. http: / / www . keynote.com.

[19] M. Marzolla and R. Mirandola. Performance prediction of web service workflows. In QoSA, volume 4880 of LNCS, pages 127-144. Springer, 2007.

[20] E. M. Maximilien and M. P. Singh. Toward autonomic web services trust and selection. In ICSOC, pages 212-221, 2004.

[21] D. Menascé. QoS issues in Web Services. IEEE Internet Comp., 6(6):72-75, 2002.

[22] OASIS. Web Services Business Process Execution Language version 2.0, Jan. 2007. http: / / www . oas is-open. org.

[23] Y. Qu, C. Lin, Y. Wang, and Z. Shan. Qos-aware composite service selection in grids. In Int'l Conf. Grid and Cooperative Computing, pages 458-465, 2006.

[24] D. Rud, A. Schmietendorf, and R. Dumke. Performance modeling of ws-bpel-based web service compositions. In IEEE Services Computing Workshops, pages 140-147, 2006.

[25] K. Verma, P. Doshi, K. Gomadam, J. A. Miller, and A. P. Sheth. Optimal adaptation in web processes with coordination constraints. In IEEE ICWS, 2006.

[26] T. Yu, Y. Zhang, and K.-J. Lin. Efficient algorithms for web services selection with end-to-end qos constraints. ACM Trans. Web, 1(1):1-26, 2007.

[27] L. Zeng, B. Benatallah, M. Dumas, J. Kalagnamam, and H. Chang. QoS-aware middleware for web services composition. IEEE Trans. Softw. Eng., 30(5), May 2004. 